\title{
The RSRF/MEF2 protein SL1 regulates cardiac muscle-specific transcription of a myosin light-chain gene in Xenopus embryos
}

\author{
Anne E. Chambers, Malcolm Logan, Surendra Kotecha, Norma Towers, Duncan Sparrow, \\ and Timothy J. Mohun ${ }^{1}$ \\ Laboratory of Developmental Biochemistry, National Institute for Medical Research, The Ridgeway, Mill Hill, \\ London NW7 1AA, UK
}

We have examined the role of two RSRF/MEF2 proteins in the onset of skeletal and cardiac muscle differentiation in early Xenopus embryos. In normal development, zygotic expression of SL1 (MEF2D) precedes that of SL2 (MEF2A) by several hours, but neither gene is expressed prior to the accumulation of MyoD and Myf5 transcripts in the somitic mesoderm. Ectopic expression of the myogenic factors in explants of presumptive ectoderm induces expression of both SL1 and SL2, whereas in reciprocal experiments, neither RSRF protein activates the endogenous myoD or Myf5 genes. We conclude that SL1 and SL2 lie downstream of these myogenic factors in the skeletal myogenic pathway. SL1 is distinguished from SL2 in being expressed in the presumptive heart region of the early tailbud embryo, prior to detection of any markers for cardiac muscle differentiation. Furthermore, ectopic SL1 induces the expression of an endogenous cardiac muscle-specific myosin light-chain (XMLC2) gene in cultured blastula animal pole explants, whereas SL2 has no comparable effect. These results demonstrate that in addition to a possible role in skeletal myogenesis, SL1 also acts in vivo as a regulator of cardiac muscle-specific transcription.

[Key Words: RSRF/MEF-2; myosin light chain; cardiac-specific transcription; Xenopus]

Received February 3, 1994; revised version accepted April 20, 1994.

The $R S R F$ (or $M E F 2$ ) genes encode a family of sequencespecific DNA-binding proteins that are thought to play an important role in the differentiation of several cell types, including muscle and neuronal tissue. Four different $R S R F$ genes have been identified so far, and their transcripts have been detected in a wide variety of vertebrate tissues and cultured cell lines (Pollock and Treisman 1991; Chambers et al. 1992; Yu et al. 1992; Breitbart et al. 1993; Leifer et al. 1993; McDermott et al. 1993). Each gene gives rise to multiple transcripts through alternate splicing, and the RSRF protein family potentially contains many distinct polypeptides. These proteins possess an amino-terminal portion that is virtually invariant throughout the family and encompasses the DNA-binding domain. The remainder of their sequence shows little conservation between family members.

RSRF proteins recognize an A/T-rich DNA sequence motif (Pollock and Treisman 1990; Chambers et al. 1992) and can bind to the target sequence either as homodimers or as heterodimers with other members of the

\footnotetext{
${ }^{1}$ Corresponding author.
}

RSRF family. A similar sequence specificity has been found for a number of previously characterized DNAbinding activities, and it is now clear that these contain RSRF proteins. One such binding activity, termed MEF2, is prevalent in muscle cells (Gossett et al. 1989). MEF2 activity increases during normal differentiation of skeletal myoblasts and is rapidly induced in fibroblasts in response to myogenic conversion by members of the MyoD family (Cseriesi and Olson 1991; Lassar et al. 1991). Sequences matching the RSRF/MEF2-binding site consensus have been found in the regulatory regions of many muscle-specific genes, and for several of these, the functional importance of the MEF2 sites has been established (Braun et al. 1989; Gossett et al. 1989; Horlick and Benfield 1989; Cserjesi and Olson 1991; Wentworth et al. 1991). MEF2 is therefore thought to be a transcriptional regulator that acts in concert with members of the MyoD family to activate skeletal myogenesis. Among its targets are the myogenin and myoD genes themselves (Edmondson et al. 1992b; Cheng et al. 1993; Yee and Rigby 1993; Leibham et al. 1994), indicating that products of the RSRF gene family play a role in the early steps of muscle differentiation.

Many other cell types also contain DNA-binding ac- 
tivities that cross-react with RSRF-specific antibodies, but little is known about their function. Several studies have described a ubiquitous MEF2-like activity (Hobson et al. 1988; Horlick et al. 1990; Pollock and Treisman 1991; Chambers et al. 1992), consistent with the detection of of RSRF transcripts in all cell types so far examined. Putative RSRF-binding sites have been identified in the control regions of several growth factor-induced genes (Pollock and Treisman 1991), raising the possibility that RSRF proteins may play a role in regulating cell proliferation. Among vertebrate tissues, RSRF transcripts are relatively prevalent in cardiac as well as skeletal muscle (Chambers et al. 1992; Navankasattusas et al. 1992; Molkentin and Markham 1993|, and MEF2-like motifs are essential for cardiocyte-restricted expression of several genes (Iannello et al. 1991; Nakatsuij et al. 1992; Navankasattusas et al. 1992; Parmacek et al. 1992; Molkentin and Markham 1993). Elevated levels of RSRF transcripts are also found in neural tissue, and expression of one RSRF gene, MEF2C, is largely restricted to cortical neurons within the mammalian brain (Leifer et al. 1993; Martin et al. 1993). MEF2-like activities may therefore be important in the differentiation of cardiac muscle and neural cell types.

Little is currently known about the precise function of individual $R S R F$ gene products or the role of variants that result from alternative splicing. In vitro, subtle differences have been detected in the binding-site preference of some RSRF proteins (Pollock and Treisman 1991), but no comparable cell type-specific variation in natural RSRF/MEF2-binding sites has been detected so far. In transfected fibroblasts, constitutive expression of $R S R F$ genes results in trans-activation of reporter genes containing synthetic RSRF/MEF2-binding sites / Yu et al. 1992; Breitbart et al. 1993; McDermott et al. 1993; Leibham et al. 1994|, but each member of the family behaves similarly in such an assay. In cultured skeletal myoblasts, members of the RSRF gene family are transcribed in a distinct temporal sequence during differentiation (Breitbart et al. 1993), suggesting that at least in these cells, individual RSRF proteins perform distinct functions.

In this study we have examined the relative roles of $S L 1$ and $S L 2$, two RSRF genes that are expressed in early embryos of the amphibian Xenopus laevis (Chambers et al. 1992). We have found previously that expression of both genes is initially restricted to the newly forming somites of the embryo and is subsequently localized to the myotomal muscle of the tadpole. Zygotic expression of $S L 1$ (the homolog of mammalian MEF2D) commences in the early gastrula, considerably before any muscle differentiation markers can be detected, whereas $S L 2$ $(M E F 2 A)$ is activated after the onset of myotomal (skeletal) muscle differentiation.

By use of microinjection of synthetic RNAs into developing embryos, we have tested the relative abilities of ectopic RSRF and MyoD proteins to activate skeletal and cardiac differentiation markers in embryo explants. Our results demonstrate that both RSRF proteins lie below $\mathrm{XMyoD}$ and XMyf5 in the skeletal muscle regulatory hierarchy. Neither RSRF protein induces the expression of skeletal muscle markers in cultured explants, but SL1 can be distinguished from SL2 by its ability to induce the expression of a myosin light-chain (MLC2) gene. In normal development, transcripts of the $M L C 2$ gene are localized exclusively to the developing heart of the tadpole and are present in both atrial and ventricular muscle. We show that $S L 1$ transcripts accumulate in the presumptive heart region of the tailbud embryo, prior to the onset of cardiac muscle differentiation. These results demonstrate that SL1 is a transcriptional regulator of cardiac muscle differentiation and provide the first direct evidence that individual members of the RSRF/MEF2 family have distinct regulatory functions in vivo.

\section{Results}

$X M y o D$ is expressed prior to the RSRF genes during gastrulation

In Xenopus embryos, the first myogenic factor to be expressed is XMyoD (Hopwood et al. 1989, 1991; Harvey 1990, 1991). Transcripts accumulate in the newly formed mesoderm of the early gastrula embryo, and $\mathrm{XMyoD}$ protein can be detected in the nuclei of the dorsal, presomitic mesoderm (Hopwood et al. 1992). In situ hybridization studies have shown that zygotic expression of the Xenopus RSRF/MEF2 gene SL1 also commences during gastrulation, and in later stages its transcripts are also localized in the somitic mesoderm (Chambers et al. 1992). The XmyoDa gene contains a MEF2-binding site coincident with the TATA motif and constitutive expression of RSRF genes activates a minimal XmyoDa promoter in COS-1 cells (Leibham et al. 1994). These results raise the possibility that RSRF proteins may be responsible for $X m y o D$ expression in the presomitic mesoderm of early gastrulae.

To investigate this possibility, we first determined the relative order of $X m y O D$ and $R S R F$ gene expresssion in synchronous embryos from blastula to neurula stages, by use of an RNase protection assay (Fig. 1). Cardiac actin transcription was monitored in the same samples, because in vertebrate embryos this gene is expressed in both cardiac and skeletal muscle. In amphibians, it provides a sensitive marker for the onset of terminal differentiation of myotomal (skeletal) muscle that forms the tadpole body musculature (Mohun et al. 1984).

$X m y o D$ transcripts were first detected in early gastrulae (stage 10.5), several hours before those of cardiac actin (Fig. 1, cf. A and C). In contrast, transcripts of the SL1 gene are present at all stages of early development (Fig. 1B). In the blastula embryo (stage 8), these derive from a maternal store, present in both oocytes and the unfertilized egg (Chambers et al. 1992). During subsequent development, the level of maternal transcripts declines, and an increase is first detected in mid-gastrulation (stage 11.5), indicating that zygotic transcription of $S L 1$ commences before that of differentiation markers such as the cardiac actin gene but after the accumulation of $X m y o D$ transcripts in the presomitic mesoderm. It also 


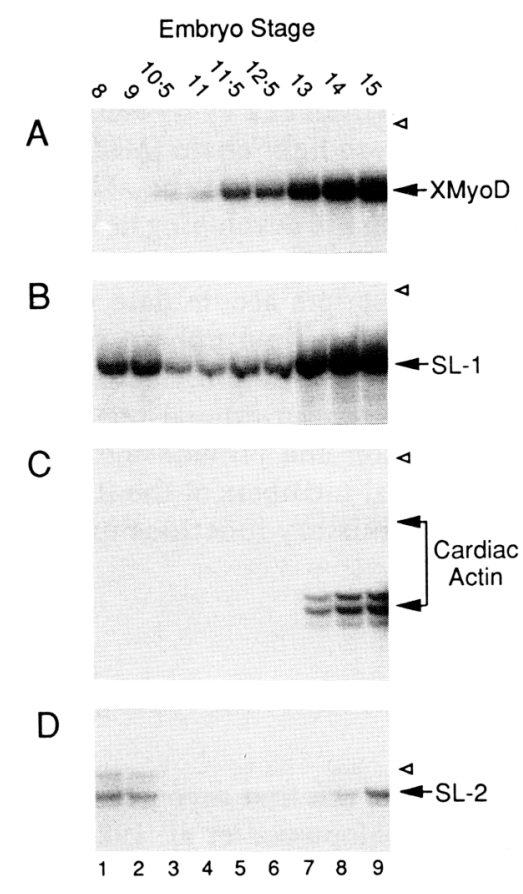

Figure 1. Sequential expression of $X m y o D$ and the $R S R F$ genes during early development. RNAs from successive stages of development from blastula (stage 8) to mid-neurula (stage 15) were analyzed by RNase protection assay for the presence of $X m y o D$ $(A), S L 1(B)$, cardiac actin $(C)$, and $S L 2(D)$ transcripts. Total RNA $(10 \mu \mathrm{g})$ was used for each assay. For each probe, the positions of protected fragments (solid arrows) and undigested probe (open triangles) are inidicated.

follows the first detection of XMyoD protein in mesodermal cell nuclei (Hopwood et al. 1992).

$S L 2$ transcripts are also inherited by the fertilized egg, but their level falls sharply between blastula and gastrula stages. However, unlike $S L 1$, accumulation of $S L 2$ mRNA does not begin until neurulation (stage 14), several hours after terminal differentiation of somitic muscle has commenced (Fig. 1, cf. C and D). This suggests that the two RSRF genes have distinct functions in the differentiation of axial (skeletal) muscle.

\section{Activation of the RSRF genes by the myogenic factors}

Ectopic expression of genes in Xenopus embryos provides a powerful assay to investigate gene function, and we have used this approach to test directly the regulatory interactions between the $R S R F$ and myoD gene families. Fertilized eggs were injected with synthetic RNAs and animal pole explants subsequently isolated from blastula (stage 8) embryos. During normal development, animal pole tissue of the blastula contributes to ectodermal and neural tissue (Keller 1975), and in explant culture it forms balls of epidermal tissue. Explants from uninjected and injected embryos were cultured until neurula stage and the expression of a variety of muscle-specific genes monitored by RNase protection assay.

Synthetic RNAs encoding the myogenic factors
MyoD, Myf5, and myogenin were injected with an equal amount of Xenopus E12 RNA to potentiate their phenotypic effects (Rashbass et al. 1992). The RSRF RNAs were injected individually, either alone or together with $X m y o D$ and $X E 12$. They were also tested as an equimolar mixture.

All three myogenic factors were equally effective in activating the cardiac actin gene (Fig. 2A, lanes 6-8), which is a direct target for trans-activation via an E-box site within its promoter (Taylor et al. 1991). Their injection also resulted in the activation of endogenous $S L 1$ and $S L 2$ expression (Fig. 2B,C, lanes 6-10). Coinjection of $S L 1$ or $S L 2$ with $X m y o D / X E 12$ had no detectable effect on the levels of cardiac actin transcripts (Fig. 2A, lanes 9, $10)$.

In reciprocal experiments, ectopic expression of SL1 or SL2 in animal pole explants had no comparable effect. Neither caused the activation of endogenous musclespecific markers, such as the cardiac actin (Fig. 2A, lanes 2-5) Xmyf5 or XmyoD genes (data not shown), nor did they activate the expression of the endogenous $R S R F$ / $M E F 2$ genes (Fig. 2B,C). Both injected RNAs could be detected at the end of the culture period (Fig. 2B, lanes 2, 5; Fig. 2C, lanes 4, 5), and their stability was comparable with that of the synthetic myogenic factor RNA /data not shown).

Taken together with the order of expression during normal development, these results demonstrate that the two Xenopus RSRF/MEF2 genes lie downstream of the myogenic factors in the regulatory hierarchy controling myotomal muscle differentiation. We found no evidence from our injection experiments for a role of these factors in activating expression of the myogenic factors, nor did they activate skeletal myogenesis in presumptive ectodermal tissue, whether injected alone or in combination with $X m y o D$ and $X E 12$.

\section{SL1 is expressed in the prospective heart region of the Xenopus embryo}

Among adult frog tissues, expression of SL1 and SL2 is relatively high in skeletal muscle, brain, and heart (Chambers et al. 1992). In the embryo, neither gene is expressed in the developing nervous system until the swimming tadpole stage and cannot therefore be involved in early stages of neural differentiation (A. Chambers and T. Mohun, unpubl.). To test whether they may play a role in cardiac myogenesis, we took advantage of the relatively late onset of cardiogenesis in amphibians compared with mammals or birds. In Xenopus, the presumptive heart region lies on the ventral side of neurula and tailbud embryos, well separated from the dorsal, axial muscle of the myotomes (Sater and Jacobson 1990). The cardiac mesoderm is specified to form muscle tissue (Sater and Jacobson 1989) even though terminal differentiation markers are not detected until the late tailbud (stage 26) (Logan and Mohun 1993).

We dissected anteroventral fragments containing the cardiac mesoderm from neurula stage onward and exam- 


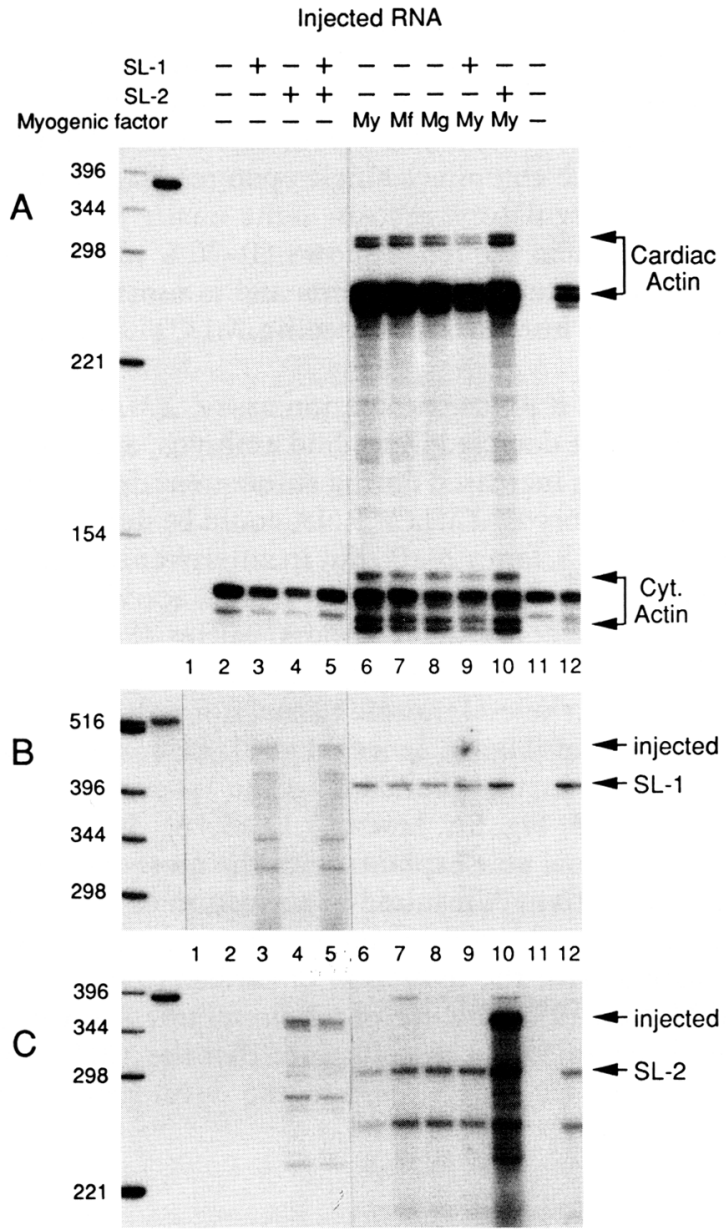

Figure 2. Ectopic expression of MyoD proteins activates RSRF gene transcription in animal pole explants. Fertilized eggs were injected with $R S R F$ and myogenic factor RNAs, cultured until blastula stage, and dissected to obtain animal pole explants. These were cultured until mid-late neurula stage and analyzed by RNase protection assay for the presence of cardiac actin $(A)$, $S L 1(B)$, and $S L 2(C)$ transcripts. (Lane 1) $10 \mu \mathrm{g}$ of tRNA (control); (lanes 2,11) uninjected; (lanes 3-5) $S L 1, S L 2$, and an equimolar combination of the two, respectively; (lanes $6-8) X E 12$ in combination with $X m y o D(M y), X m y f 5(\mathrm{Mj})$ and mouse myogenin $(\mathrm{Mg}$ ), respectively; (lanes 9,10) XE12/XmyoD with $S L 1$ and $S L 2$, respectively; (lane 12) total RNA from neurula (stage 18) embryos. The equivalent of five explants was analyzed in each assay. Approximate size markers were provided by an endlabeled HinfI digest of pBR 322 and are shown adjacent to undigested probe. Full-length protected fragments are indicated. The cardiac actin probe cross-hybridizes with ubiquitous cytoskeletal actin transcripts that give rise to a cluster of partial protection products (indicated in $A$ ). In $B$ and $C$, the presence of the injected, synthetic RNA gives rise to a protected band that is larger than that obtained from the endogenous RSRF mRNA. (A background smear was also observed routinely with injected samples). The $S L 2$ probe detects a second endogenous mRNA that is coexpressed with $S L 2$ and produces a smaller protected fragment.

ined them for the expression of the SL1 and SL2 genes. The same samples were also tested for the presence of skeletal and cardiac muscle-specific transcripts. As a control, equivalent dorsal axial fragments were removed from the same embryos and analyzed in parallel (Fig. 3).

To test whether ventral explants were contaminated with any myotome-containing tissue, all fragments were assayed for the presence of myotome-specific markers. No XmyoD RNA was detected in any ventral fragment (data not shown). However, in normal development $X m y o D$ expression declines sharply during tailbud stages and no longer provides a sensitive marker for axial muscle (Hopwood et al. 1989). We therefore tested for a second myotome-specific marker, which is detected as a partial protection product in assays for $X M H C \alpha$ and probably encodes a myotomal-specific myosin heavy chain (MHC) isoform (Logan and Mohun 1993). No signal was detected in any ventral explant, whereas the level in dorsal pieces increased dramatically in tailbud stages (Fig. 3E).

Cardiac actin mRNA was highly abundant in dorsal fragments at all stages between neurula (stage 18) and late tailbud (stage 28), owing to the presence of differentiated myotomal muscle tissue (Fig. 3A). Transcripts were first detected in ventral explants from stage 25 tailbud embryos (Fig. 3B), coincident with the appearance of myosin light chain 2 (XMLC2) mRNA in this region of the embryo. Expression of the cardiac actin and XMLC2 genes marks the onset of cardiac muscle differentiation in the embryo (see below).

Both $S L 1$ and $S L 2$ were expressed in dorsal explants, consistent with the somite and myotome-specific localization of transcripts detected in whole-mount in situ

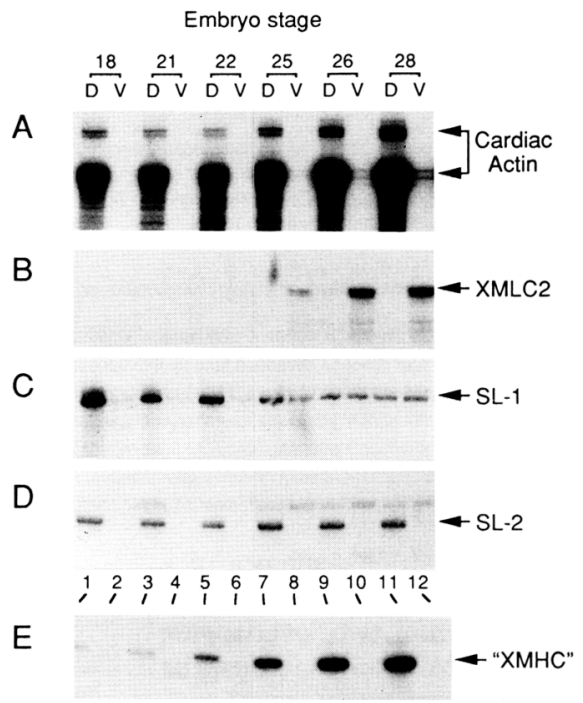

Figure 3. SL1 expression in the prospective heart region of tailbud embryos. RNA from anterior-dorsal (D) and anterior-ventral (V) fragments of embryos was analyzed by RNase protection assay for the presence of cardiac actin, $X M L C 2, S L-1$, and $S L-2$ transcripts $(A-D$, respectively). The equivalent of five fragments was used in each assay. Samples were also tested for the presence of a myotomal muscle-specific marker (labeled "XMHC") (Logan and Mohun 1993), detected using a probe for $X M H C \alpha$ RNA $(E)$. Explants were dissected from late neurula (stage 18) to late tailbud (stage 28) embryos. 
hybridization studies. SL1 transcripts show a marked gradient of expression, concentrated in more recently formed, posterior somites (Chambers et al. 1992). This accounts for the progressive decline in their abundance in anterior, dorsal explants during development (Fig. 3C). From the onset of cardiac muscle differentiation (stage 25), SL1 expression was evident in ventral explants and in longer exposures, transcripts could be detected as early as stage 21 . In contrast, SL2 mRNA was entirely restricted to dorsal explants and accumulated steadily between neurula and tailbud stages (Fig. 3D).

The two Xenopus RSRF genes therefore showed distinct temporal and spatial patterns of expression in the muscle of early embryos. Although both were expressed during skeletal myogenesis, the $S L 1$ gene was also activated in the presumptive heart region, prior to the onset of cardiac muscle differentiation.

\section{$\mathrm{XMLC2}$ is a marker for cardiac muscle differentiation}

Because $S L 1$ was expressed in the region comprising the heart anlagen, we wished to test whether ectopic expression of the Xenopus RSRF genes had any effect on genes expressed in cardiac myogenesis. A potential target for regulation by RSRF/MEF2 factors is the $M L C 2$ gene. In mammals, this gene contains an $\mathrm{A} / \mathrm{T}$-rich motif within its promoter that is essential for transcription in transfected primary cardiocytes (Navankasattusas et al. 1992). To obtain a specific probe for the Xenopus $M L C 2$ gene, we constructed an adult heart cDNA library and screened it with a probe from the human $M L C 2 a$ cDNA (Hailstones et al. 1992). A composite, full-length cDNA sequence derived from this screen is shown in Figure 4A. This sequence contains a single open reading frame that encodes a regulatory myosin light chain polypeptide. The amino acid sequence shows $60-70 \%$ identity with other vertebrate MLC2 proteins and is most closely related to the human atrial-specific MLC2 isoform (Fig. 4B).

By use of an RNase protection assay, XMLC2 expression was first detected in tailbud embryos, and the level of transcripts increased during subsequent development. In whole embryos, $X M L C 2$ RNA could be detected from stage 26 (Fig. 5, lanes 2-10), but in anteroventral explants containing the prospective heart region, activation of the gene was detected a few hours earlier (Fig. 3B). No $X M L C 2$ transcripts were detected in dorsal axial tissue (enriched for skeletal muscle tissue) nor in adult skeletal muscle tissue (Fig. 5, lanes 11, 17). In contrast, transcripts were highly abundant in the tadpole and adult heart (Fig. 3B; Fig. 5A, lanes 11,12,15,16). Treatment of blastula animal pole explants with the mesoderm-inducing factor activin A resulted in activation of the XMLC2 gene in a dose-dependent manner (Fig. 5A, lanes 13,14), consistent with the induction of cardiac muscle (Logan and Mohun 1993). By use of whole-mount RNA in situ hybridization (Fig. 6) we confirmed that the $X M L C 2$ gene is expressed exclusively within the developing tadpole

A

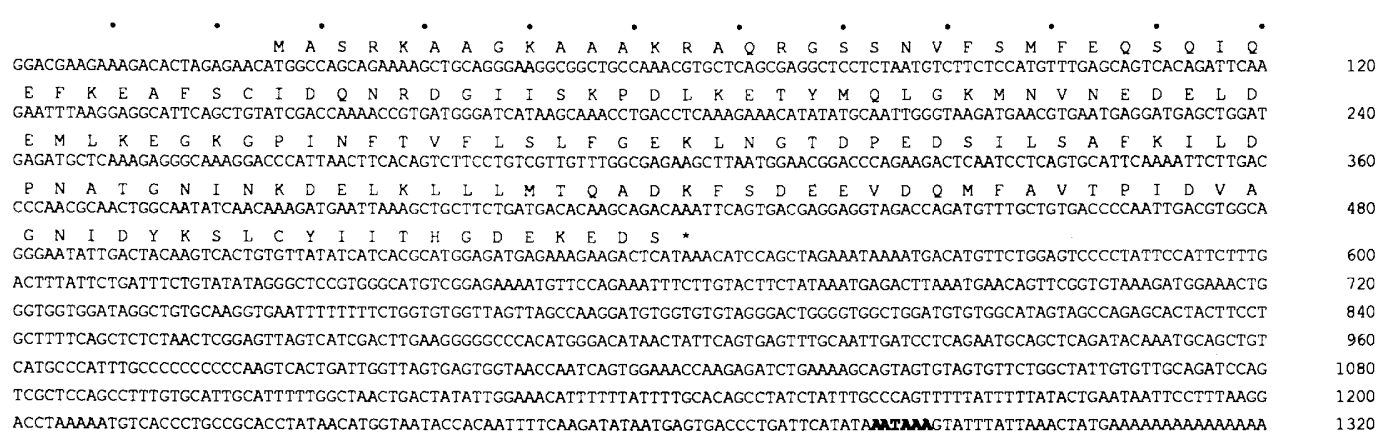

Figure 4. (A) Nucleotide sequence of a Xenopus $M L C 2$ cDNA. The nucleotide sequence is derived from two, overlapping cDNA isolates. $\lambda X M L C 2-5$ contained the entire sequence shown except exon 5 (nucleotides 393-441). This was present in a partial cDNA, $\lambda$ XMLC2-12, which lacks the first 260 nucleotides of the sequence. The predicted XMLC2 polypeptide sequence is shown, as is a putative poly $(\mathrm{A})$ addition sequence (bold). (B) Comparison

B

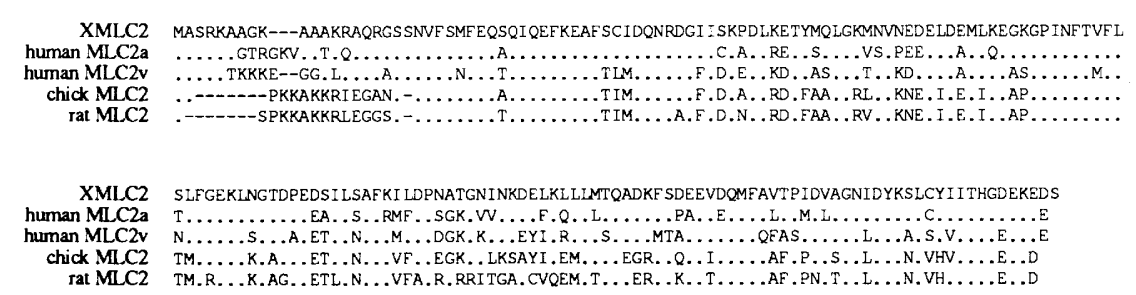

of XMLC2 and other vertebrate MLC2 proteins. The XMLC2 polypeptide sequence is aligned with the human atrial (Hailstones et al. 1992), human ventricular (EMBL accession S22101), chick (Winter et al. 1985), and rat (Henderson et al. 1989) MLC2 sequences. These are arranged in order of similarity to the frog sequence as determined by use of the UWGCG Pileup program. Only residues that differ from the frog sequence have been shown, and gaps (dashes) have been introduced to permit optimal alignment. 


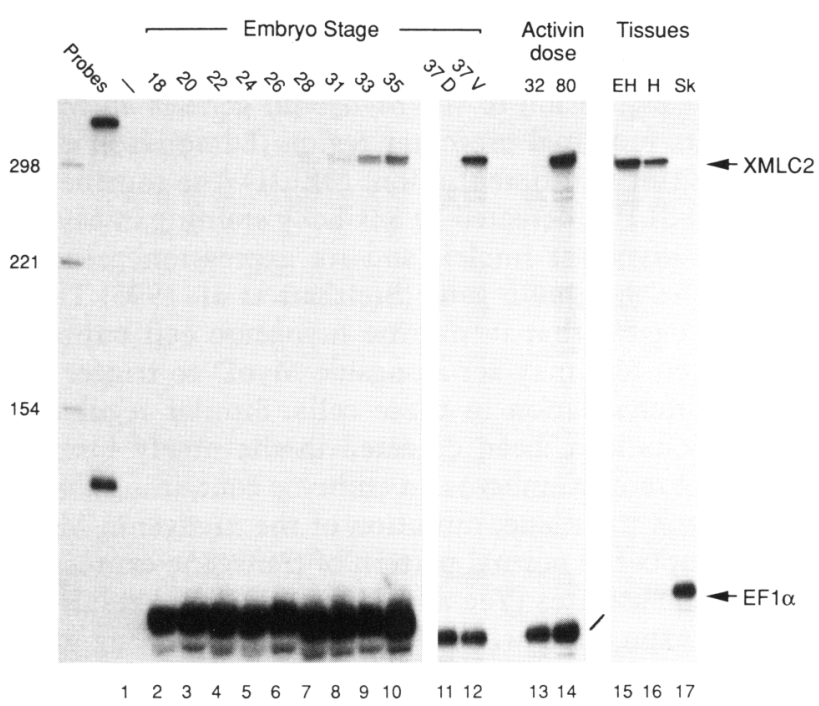

Figure 5. $X M L C 2$ expression during development. Total RNA from embryos, cultured explants, and tissues was tested for $X M L C 2$ mRNA by use of an RNase protection assay. (Lane 1) tRNA control; (lanes 2-10) two embryos from neurula to tadpole stages of development (stages 18-35, as indicated); (lanes 11,12 ) five anterior-dorsal (D) and anterior-ventral (V) regions of swimming tadpoles (stage 37); (lanes 13,14) the equivalent of five blastula animal pole explants treated with 32 or $80 \mathrm{U} / \mathrm{ml}$ of activin $A$ and cultured until stage 42; (lanes 15-17) two tadpole (stage 42 ) heart tubes (EH), $0.5 \mu \mathrm{g}$ of adult heart $(\mathrm{H}$ ), and $10 \mu \mathrm{g}$ of adult skeletal muscle (Sk). As an internal control, a probe for EF1 $\alpha$ mRNA was included in each assay. An end-labeled Hinfl digest of pBR322 was used for approximate size markers.

heart and was entirely absent from any skeletal muscle tissue at all embryonic stages examined.

\section{XMLC2 expression is activated by SL1 in embryo explants}

These results demonstrate that $X M L C 2$ mRNA provides a tissue-specific marker for cardiac muscle in Xenopus embryos. We therefore tested whether XMLC2 expression could be activated by ectopic expression of the $R S R F / M E F 2$ genes in blastula animal pole explants. High levels of $X M L C 2$ RNA were found in explants from SL1-injected embryos but were undetectable in those from uninjected siblings (Fig. 7, cf. lanes 2 and 3). Interestingly, because the cultured explants were harvested when control embryos reached mid- to late-neurula stage, SL1-induced XMLC2 transcription occurred many hours before the onset of cardiac muscle differentiation in uninjected embryos (Fig. 7, cf. lanes 3 and 5 with lanes 6 and 7). Neither the cardiac actin nor the cardiac muscle-specific $X M H C \alpha$ gene was activated in SL1-injected explant tissue (Fig. 2; data not shown), indicating that $X M L C 2$ expression was unlikely to be the result of cardiomyogenic conversion in this tissue. Rather, the $X M L C 2$ gene is probably a direct target for trans-activation by the RSRF protein.

SL2 RNA consistently failed to elicit comparable
$X M L C 2$ expression despite the abundance of SL2 protein in explants from injected embryos (data not shown). In most experiments, $X M L C 2$ transcripts were entirely undetectable in the explants (Fig. 6, lane 4). Low level expression was occasionally observed, but this corresponded to $<10 \%$ of that obtained with SL1-injected embryos. In coinjection experiments, SL2 RNA neither potentiated nor inhibited the effect of SL1 RNA (Fig. 7, cf. lanes 3 and 5). Together, these results demonstrate that despite the near identity of their binding site preferences in vitro (Pollock and Treisman 1991; Chambers et al. 1992), the two Xenopus RSRF/MEF2 proteins can be distinguished by a functional assay in vivo.

\section{Discussion}

In this study we have examined the relative roles of two $R S R F / M E F 2$ genes, $S L 1$ and $S L 2$, which are expressed during early development in Xenopus. Three main conclusions can be drawn from our experiments. First, both genes lie beneath the myogenic factors in the muscle regulatory hierarchy. Second, the two genes can be distinguished not only by the timing of their activation in the somitic mesoderm, but also by their spatial patterns of expression after neurulation. The SL1 gene is activated in the presumptive heart region of the neurula embryo, and its product acts as a transcriptional regulator of

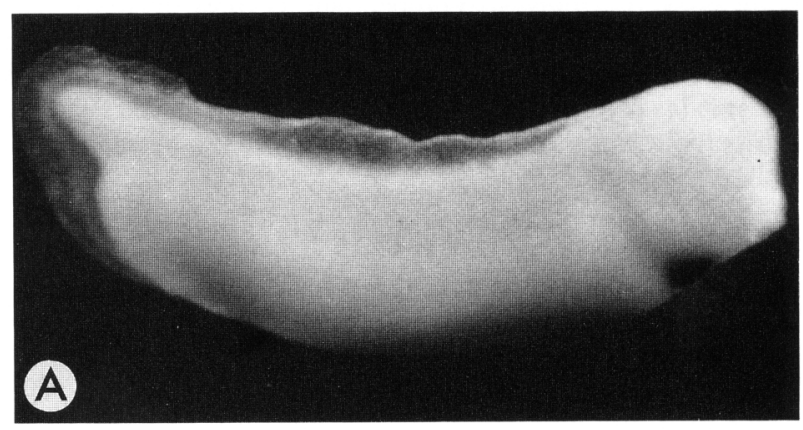

B

Figure 6. $X M L C 2$ is a marker for terminal differentiation of cardiac muscle in Xenopus embryos. The distribution of $X M L C 2$ RNA was examined by use of albino embryos and digoxigenin-labeled probes. Staining was detected first in stage $28 / 29$ tadpoles in the presumptive heart region and was subsequently confined to the developing heart tube. A stage $32 \mathrm{em}$ bryo is shown before $(a)$ and after $(b)$ clearing. In later stages, staining was detected in both atrial and venticular chambers of the beating tadpole heart. No staining was detected with a sense control probe. 


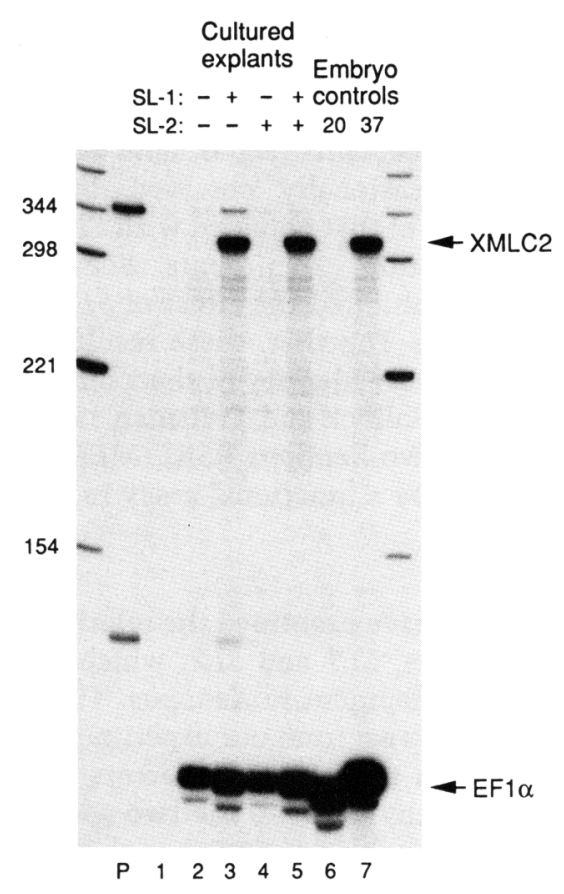

Figure 7. Ectopic expression of SL1 activates XMLC2 gene transcription in animal pole explants. Cultured animal pole explants from injected embryos were analyzed by RNase protection at the neurula stage for expression of the heart-specific $X M L C 2$ gene. Levels of $E F 1 \alpha$ mRNA were monitored in the same assay. (P) Undigested probe; (lane 1) $10 \mu \mathrm{g}$ of tRNA; (lane 2) control explants (uninjected); (lanes 3-5) explants from embryos injected with SL1 and SL2 RNA, individually or in combination (as indicated); (lanes 6,7) $5 \mu \mathrm{g}$ of late neurula (stage 20) and tadpole (stage 37) RNA, respectively. (Size markers as in earlier figures).

the cardiomyogenic program. Last, the cardiac musclespecific $X M L C 2$ gene is a target for trans-activation by SL1 but not SL2 protein, demonstrating that individual RSRF proteins possess distinct functions in vivo.

\section{RSRF/MEF2 genes in skeletal myogenesis}

In skeletal myoblasts, MEF2 activity is detected only after expression of MyoD family members. The same activity is rapidly induced in fibroblasts by constitutive expression of the myogenic factors (Lassar et al. 1991), even when the cells are incapable of terminal muscle differentiation (Cserjesi and Olson 1991). This suggests that one or more of the MyoD protein family directly activate the $R S R F / M E F 2$ genes. Taken together with the identification of potential MEF2-binding sites in the regulatory regions of many muscle-specific genes, these results have led to a model in which the RSRF/MEF2 genes lie downstream of the myogenic factors and coordinate activation of MEF2-regulated, skeletal muscle genes $[\mathrm{Ol}$ son 1992).

There is also evidence to suggest that MEF2-like proteins regulate expression of the myogenic factors themselves and thus mediate a positive feedback loop that may be important in establishing the myogenic phenotype. In myotube cultures, an important regulatory element for expression of the myogenin gene is an MEF2 site in its proximal promoter region (Edmondson et al. 1992a). Prior to differentiation, MEF2D (the murine homolog of SL1) is detected by antibody staining in MyoDpositive myoblast nuclei, and its expression precedes that of the myogenin gene (Breitbart et al. 1993) These results suggest that in murine myogenic cell cultures, MEF2D protein may act alongside MyoD to trigger terminal differentiation in these cells. Similar regulatory interactions have been detected in the newly forming muscle of mouse embryos. In embryos containing a myogenin-lacZ transgene, mutation of the myogenin MEF2 site disrupts the normal pattern of transgene expression within the somites (Yee and Rigby 1993) and results in delayed expression within the limb buds (Cheng et al. 1993).

In cultured cell lines, the onset of myogenic differentiation is signaled by myogenin expression, and an essential role for this protein is confirmed by studies of transgenic mice lacking the myogenin gene (Hasty et al. 1993). If RSRF/MEF2 proteins activate myogenin expression, we might expect that overexpression of $R S R F$ / $M E F 2$ genes in cell lines may trigger myogenic differentiation. However, in Xenopus embryos, myogenesis occurs without any detectable myogenin expression, and for RSRF proteins to play a similar role, they must activate expression of XmyoD and/or Xmyf5. A MEF2 site has been identified in the Xenopus myoD gene promoter (Leibham et al. 1994), but the role of this motif in normal myotome differentiation is unknown.

Zygotic expression of the RSRF genes within the somitic mesoderm only appears to commence after expression of $X m y o D$ and $X m y f 5$, indicating that the rapid accumulation of RSRF/MEF2 factors in the somitic mesoderm is unlikely to be responsible for initial activation of the myogenic regulators. As in cultured skeletal myoblasts, $S L 1(M E F 2 D)$ is expressed prior to muscle-specific markers, whereas $S L 2(M E F 2 A)$ is expressed only after terminal differentiation has begun. We cannot exclude a role for maternally inherited SL1 or SL2 protein in the onset of $X m y o D$ and $X m y f 5$ expression, but if this is the case, there must also be mechanisms to restrict their activity to the presomitic mesoderm because these factors are distributed throughout the early embryo. In a direct test of their roles, ectopic expression of SL1 and $S L 2$ failed to activate the endogenous $X m y o D$ and $X m y f 5$ genes in animal pole tissue, whereas both $R S R F$ genes were activated by ectopic expression of Xenopus and murine myo $D$ family members. We do not think this difference results from insufficient accumulation of RSRF protein in the animal pole cells of $S L 1$ - or SL2-injected embryos because both proteins are readily detected in the explants and their level is manyfold greater than the level of maternal RSRF protein in normal embryos (D. Sparrow and T. Mohun, unpubl.). These results argue strongly that factors other than SL1 or SL2 activate the $X m y o D$ and $X m y f 5$ genes in vivo. Our experiments do not exclude other roles for the RSRF/MEF2 proteins in 
regulating expression of the myoD family. It is entirely possible that the accumulating SLl and SL2 proteins act to maintain or indeed amplify expression of $X m y o D$ and Xmyf5 within the somitic mesoderm.

In Xenopus embryos, ectopic expression of myogenic factors is insufficient to induce muscle formation in the prospective ectodermal tissue derived from animal pole explants (Hopwood and Gurdon 1990). It does, however, result in the activation of E-box-regulated genes (such as cardiac actin), and this effect is potentiated by coexpression of XE12 (Rashbass et al. 1992). We interpret this to mean that endogenous genes that are activated by exogenous myogenic factors constitute direct trans-activation targets for these proteins. On this basis, it is likely that muscle-specific expression of the RSRF genes is attributable to the presence of E-box sites within their regulatory regions. In Xenopus, these would be binding sites for XMyoD and/or XMyf5 because XMRF4 is only expressed later in development (Jennings 1992). Interestingly, ectopically expressed mouse myogenin is as effective in trans-activating the $R S R F$ and cardiac actin genes as the other myogenic factors, even though the Xenopus myogenin gene is not apparently expressed at any stage. We do not know whether this indicates a limitation in the usefulness of the ectopic expression assay or simply testifies to functional redundancy among members of the MyoD family.

\section{$R S R F / M E F 2$ in cardiac myogenesis}

The possibility that RSRFs may play a role in cardiac myogenesis was first suggested by the studies of Chien and co-workers who identifed an RSRF/MEF2-like binding site essential for expression of the rat $M L C 2$ gene in primary cardiocytes (Zhu et al. 1991; Navankasattusas et al. 1992). Similar sequences are required for cardiac muscle-specific expression of the chick cardiac troponin $\mathrm{T}$ (Iannello et al. 1991), mouse troponin C (Parmacek et al. 1992), rat MHC $\alpha$ (Molkentin and Markham 1993), and human phosphoglycerate mutase (Nakatsuji et al. 1992) genes. RSRF/MEF2-binding activity is prevalent cardiac muscle cell extracts (Chambers et al. 1992; Navankasattusas et al. 1992; Molkentin and Markham 1993), and immunofluorescence studies have identified RSRF/ MEF2 proteins in nuclei of primary cardiocytes (Breitbart et al. 1993).

We have found that during normal development, SL1 mRNA accumulates in an anteroventral region of the Xenopus neurula embryo. This encompasses the cardiac mesoderm, which only differentiates several hours later. In functional assays, ectopic expression of SL1 in explants of presumptive ectoderm causes activation of the cardiac muscle-specific $M L C 2$ gene. Together, these results demonstrate unequivocally that SLl is an early regulator of cardiac muscle differentiation.

The XMLC2 gene that we have identified is most closely related in sequence to the human $M L C 2 a$ gene, about which little is known (Hailstones et al. 1992). $M L C 2 a$ expression is restricted exclusively to fetal atrial muscle, whereas the XMLC2 transcripts are abundant in cardiac tissue throughout development and are present in both atrial and ventricular muscle (T. Mohun, unpubl.). XMLC2 activation is not the result of cardiomyogenic conversion of ectodermal tissue because neither of the other two markers for cardiac muscle (cardiac actin and $X M H C \alpha$ genes) is expressed in the explants. This specificity suggests that the $X M L C 2$ gene is a direct target for trans-activation by the SL1 protein. Preliminary studies of the XMLC2 gene promoter have identified two adjacent motifs that match the RSRF/MEF2 consensusbinding site, but their functional role has yet to be tested. The absence of any XMHC $\alpha$ expression in SL1injected explants is surprising because cardiocyte-specific expression of the rat homolog is dependent on a MEF2-binding site in its promoter (Molkentin and Markham 1993). Other proteins in addition to RSRFs have been identified that bind to MEF2-like sequences (Cserjesi et al. 1992; Zhou et al. 1993; Zhu et al. 1993), and it is possible that one of these regulates the $M H C \alpha$ gene. Alternatively, cardiac-specific expression of the Xenopus $M H C \alpha$ gene may result from other regulatory mechanisms.

In earlier studies, we found that maternally derived RSRF/MEF2 activity declines during early development and is undetectable in animal pole explants after overnight culture. (Chambers et al. 1992). Ectopic expression of $S L 1$ in such explants activates the XMLC2 gene but does not similarly activate the $S L 2$ gene. The XMLC2 gene must therefore be trans-activated either by an SLl homodimer or by SL1 in association with another partner that is normally present in presumptive ectodermal cells. In either case, it is intriguing that the XMLC2 gene is expressed only in cardiac muscle, because in the early embryo, SL1 is expressed at high levels in somitic muscle in early embryos. One possibility is that somitic mesoderm contains a negative regulator (perhaps induced by XmyoD or XMyf5) that blocks XMLC2 gene activation during skeletal muscle differentiation. In adult tissues, analogous mechanisms must exist to restrict $X M L C 2$ expression because $S L 1$ is widely expressed and is relatively abundant in both spleen and brain.

\section{Specificity of RSRF function}

In transfection studies, all members of the RSRF/MEF2 family trans-activated reporter genes containing a pair of synthetic binding sites adjacent to a basal promoter. Furthermore, similar activity was obtained with all of the splice variants examined. This approach is therefore of little use in assessing the individual roles of each RSRF/ MEF2 protein. In contrast, ectopic expression in embryo explants provides an assay that can reveal functional differences between the SL1 and SL2 proteins. It will therefore be useful in identifying the basis for specificity of RSRF/MEF2 protein function.

SL1 and SL2 proteins differ in only 8 of 91 residues across their DNA-binding domains, and their consensusbinding sites in vitro are virtually identical. For this reason it is puzzling that coexpression of $S L 2$ had no effect on the activity of $S L 1$. If both proteins bind similar sites 
in vivo, we might expect that SL2 would act as competitive inhibitor of SL1, blocking activation of the XMLC2 gene. Alternatively, interaction of SLl with other factors on the $X M L C 2$ promoter might stabilize binding or produce a complex with higher affinity for the binding site. Characterization the XMLC2 promoter should clarify this issue.

\section{Materials and methods \\ Embryo culture, dissection, and injection}

Synchronous embryos were obtained by artificial fertilization and cultured in $0.1 \times \mathrm{NAM}$ at $18-21^{\circ} \mathrm{C}$. These were staged according to Nieuwkoop and Faber (1956). Blastula animal pole explants were dissected from stage 8 embryos and cultured in $3 / 4 \times$ NAM, in the presence or absence of activin A. Dorsal and ventral explants were dissected from neurula and tailbud embryos as described previously (Logan and Mohun 1993). For RNA injections, $\sim 5 \mathrm{ng}$ of synthetic RNA was injected into fertilized eggs at the one- or two-cell stage, with an injection volume of 8-12 nl per blastomere. Injections were carried out in $3 / 4 \times$ NAM containing $3 \%$ Ficoll, and embryos were transferred to $3 / 4 \times$ NAM after $\sim 1 \mathrm{hr}$.

\section{RNA synthesis in vitro}

Fragments containing the entire $S L 1$ - and $S L 2$-coding sequences (nucleotides 235-1968 and nucleotides 205-1920, respectively; Chambers et al. 1992) were prepared by PCR and cloned via $\mathrm{Ncol}$ ends into the transcription vector $\mathrm{pSP} 64 \mathrm{~T}(\mathrm{Nco})$ to produce pSL1.64T and pSL2.64T. The resulting plasmids contain the RSRF-coding sequence flanked by the human $\beta$-globin leader and a portion of the Xenopus $\beta$-globin 3'-untranslated region. Templates for RNA synthesis were prepared by linearization with EcoRI. The mouse myogenin-coding sequence was also amplified by PCR and cloned via linkers as a BgIII-BamHI fragment into the transcription vector pSP64T (Melton et al. 1985) to give pMg.64T. The DNA sequence of each construct was verified by use of specific primers. Capped RNA for injection into eggs was synthesized in vitro (Krieg and Melton 1984) with SP6 RNA polymerase. XmyoD, XMyf5, and XE12 RNA were prepared similarly with $X$ hol-linearized templates from pSP64T-XmyoD (Hopwood and Gurdon 1990), pSP64T-XMyf5 (Hopwood et al. 1991), and pSP64-XE12ßM (Rashbass et al. 1992). Several different preparations of each synthetic RNA were used for embryo injection experiments.

\section{RNA preparation and assay}

RNA was prepared from embryos and adult tissue as described previously (Mohun et al. 1984). The following templates were used to prepare RNA probes for use in RNase protection assays. For XmyoD: pSP73-5bG/XmyoD comprises a 295-nucleotide HindIII-Sall fragment of pSP64T-XmyoD subcloned into pSP73. An RNA probe was prepared with SP6 RNA polymerase and Xhol-linearized template DNA. For $S L 1$ and $S L 2$ : pSP73.SL-1 and pKS.SL-2 $\Delta 71$ (Chambers et al. 1992) were used in Figures 1 and 2. To distinguish endogenous from synthetic RNAs (Fig. 3), templates were prepared from plasmids pSP73.SL1 (64T) and pSP65.SL2(64T) by linearization with HindIII. These were derived from pSL1.64T and pSL2.64T and contain the entire human $\beta$-globin $5^{\prime}$-untranslated region fused to 405 and 304 nucleotides of the $S L 1$ - and $S L 2$-coding sequences, respectively. Templates for cardiac actin $(p S P \alpha 1)$ (Mohun et al.
1988), XMHC $\alpha$ (pXMHC $\alpha 1 / 3$ ) (Logan and Mohun 1993), and $E F 1 \alpha$ (Sargent and Bennett 1990) probes have been described previously. $X M L C 2$ transcripts were detected with a probe prepared from pXMLC2 $\Delta$ spanning nucleotides 1-309 of the $X M L C 2$ cDNA sequence. This was synthesized using EcoRIlinearized template DNA and T7 RNA polymerase.

\section{Whole-mount RNA in situ hybridization}

Albino embryos (stages 22-46) were used for whole-mount in situ hybridization as described by Harland (1991) using digoxigenin-labeled probes. The antisense probe was identical to that used for RNase protection assays (see above). A sense control probe was synthesized from HindIII-linearized pXMLC2 $\Delta$ template by use of T3 RNA polymerase.

\section{Isolation of XMLC2 $c D N A$}

A directional cDNA library was created by use of the UniZapII kit (Stratagene) and screened with an oligo-labeled probe containing the entire coding region of the human $M L C 2 a$ cDNA (Hailstones et al. 1992). Several cDNA positively hybridizing clones were analyzed and found to contain overlapping regions of a Xenopus MLC2 cDNA. pXMLC2-5 consisted of an apparently full-length cDNA that subsequently proved to be a splice variant in which exon 5 is absent, resulting in a frameshift mutation. pXMLC2-12 consisted of a partial cDNA derived from the same gene and including exon 5 (see Fig. 4A). The entire $X M L C 2$ cDNA sequence was constructed as a composite from the two cDNAs, which were sequenced by the shotgun procedure. The composite sequence was analyzed using the STADEN (Staden 1982, 1984) and UWGCG (Devereux et al. 1984) programs.

\section{Acknowledgments}

We thank Robert Wilson and Peter Rigby for helpful discussions and critical review of the manuscript.

\section{References}

Braun, T., E. Tannich, G. Buschhausen-Denker, and H. Arnold. 1989. Promoter upstream elements of the chicken cardiac myosin light-chain 2-A gene interact with trans-acting regulatory factors for muscle-specific transcription. Mol. Cell. Biol. 9: 2513-2525.

Breitbart, R.E., C.-S. Liang, L.B. Smoot, D.A. Laheru, V. Mahdavi, and B. Nadal-Ginard. 1993. A fourth human MEF2 transcription factor, hMEF2D, is an early marker of the myogenic lineage. Development 118: 1095-1106.

Chambers, A.E., S. Kotecha, N. Towers, and T.J. Mohun. 1992. Muscle-specific expression of SRF-related genes in the early embryo of Xenopus laevis. EMBO I. 11: 4981-4991.

Cheng, T.C., M.C. Wallace, J.P. Merlie, and E.N. Olson. 1993. Separable regulatory elements governing myogenin transcription in mouse embryogenesis. Science 261: 215--218.

Cserjesi, P. and E.N. Olson. 1991. Myogenin induces the myocyte-specific enhancer binding factor MEF-2 independently of other muscle-specific gene products. Mol. Cell. Biol. 11: 4854-4862.

Cserjesi, P., B. Lilly, L. Bryson, Y. Wang, D. Sassoon, and E.N. Olson. 1992. Mhox: A mesodermally restricted homeodomain protein that binds an esssential site in the muscle creatine kinase enhancer. Development 115: 1087-1101.

Devereux, J., P. Haeberli, and O. Smithies. 1984. A comprehen- 
sive set of sequence analysis programs for the VAX. Nucleic Acids Res. 12: 387-395.

Edmondson, D.G., T. Cheng, P. Cserjesi, T. Chakraborty, and E.N. Olson. 1992a. Analysis of the myogenin promoter reveals an indirect pathway for positive autoregulation mediated by the muscle-specific enhancer factor, MEF-2. Mol. Cell. Biol. 12: 3665-3677.

- 1992b. Analysis of the myogenin promoter reveals an indirect pathway for positive autoregulation mediated by the muscle-specific enhancer factor MEF-2. Mol. Cell. Biol. 12: 3665-3677.

Gossett, L.A., D.J. Kelvin, E.A. Sternberg, and E.N. Olson. 1989. A new myocyte-specific enhancer-binding factor that recognizes a conserved element associated with multiple musclespecific genes. Mol. Cell. Biol.. 9: 5022-5033.

Hailstones, D., P. Barton, P. Chan-Thomas, S. Sasse, C. Sutherland, E. Hardeman, and P. Gunning. 1992. Differential regulation of the atrial isoforms of the myosin light chains during striated muscle development. J. Biol. Chem. 267: 2329523300.

Harland, R.M. 1991. In situ hybridisation: An improved whole mount method for Xenopus embryos (ed. B.K. Kay and H.B. Peng, pp. 685-695. In Xenopus laevis: Practical uses in cell and molecular biology, Academic Press, London, UK.

Harvey, R.P. 1990. The Xenopus MyoD gene: An unlocalised maternal mRNA predates lineage-restricted expression in the early embryo. Development 108: 669-680.

- 1991. Widespread expression of MyoD genes in Xenopus embryos is amplified in presumptive muscle as a delayed response to mesoderm induction. Proc. Natl. Acad. Sci. 88: 9198-9202.

Hasty, P., A. Bradley, J.H. Morris, D.G. Edmondson, J.M. Venuti, E.N. Olson, and W.H. Klein. 1993. Muscle deficiency and neonatal death in mice with a targeted mutation in the myogenin gene. Nature 364: 501-506.

Henderson, S.A., M. Spencer, A. Sen, C. Kumar, M.A. Siddiqui, and K.R. Chien. 1989. Structure, organization, and expression of the rat cardiac myosin light chain-2 gene. Identification of a 250-base pair fragment which confers cardiac-specific expression. J. Biol. Chem. 264: 18142-18148.

Hobson, G.M., M.T. Mitchell, G.R. Molloy, M.L. Pearson, and P.A. Benfield. 1988. Identification of a novel TA-rich DNA binding protein that recognizes a TATA sequence within the brain creatine kinase promoter. Nucleic Acids Res. 16: 8925-8944.

Hopwood, N.D. and J.B. Gurdon. 1990. Activation of muscle genes without myogenesis by ectopic expression of MyoD in frog embryo cells. Nature 347: 197-200.

Hopwood, N.D., A. Pluck, and J.B. Gurdon. 1989. MyoD expression in the forming somites is an early response to mesoderm induction in Xenopus embryos. EMBO $/$. 8: 34093417.

- 1991. Xenopus Myf-5 marks early muscle cells and can activate muscle genes ectopically in early embryos. Development 111: 551-560.

Hopwood, N.D., A. Pluck, J.B. Gurdon, and S.M. Dilworth. 1992. Expression of XmyoD protein in early Xenopus laevis embryos. Development 114: 31-38.

Horlick, R.A. and P.A. Benfield. 1989. The upstream musclespecific enhancer of the rat muscle creatine kinase gene is composed of multiple elements. Mol. Cell. Biol.. 9: 23962413.

Horlick, R.A., G.M. Hobson, J.H. Patterson, M.T. Mitchell, and P.A. Benfield. 1990. Brain and muscle creatine kinase genes contain common TA-rich recognition protein-binding regulatory elements. Mol. Cell. Biol. 10: 4826-4836.
Iannello, R.C., J.H. Mar, and C.P. Ordahl. 1991. Characterization of a promoter element required for transcription in myocardial cells. J. Biol. Chem. 266: 3309-3316.

Jennings, C.G. 1992. Expression of the myogenic gene MRF4 during Xenopus development. Dev. Biol. 150: 121-132.

Keller, R.E. 1975. Vital dye mapping of the gastrula and neurula of Xenopus laevis. I. Prospective areas and morphogenetic movements of the superficial layer. Dev. Biol. 42: 222-241.

Krieg, P.A. and D.A. Melton. 1984. Functional messenger RNAs are produced by SP6 in vitro transcription of cloned cDNAs. Nucleic Acids Res. 12: 7057-7070.

Lassar, A.B., R.L. Davis, W.E. Wright, T. Kadesch, C. Murre, A. Voronova, D. Baltimore, and H. Weintraub. 1991. Functional activity of myogenic HLH proteins requires hetero-oligomerization with E12/E47-like proteins in vivo. Cell 66: 305315.

Leibham, D., M. Wong, T. Cheng, S. Schroeder, A.P. Weil, E.N. Olson, and M. Perry. 1994. Binding of TFIID and MEF2 to the TATA element activates transcription of the Xenopus $M y$ oDa promoter. Mol. Cell. Biol. 14: 686-699.

Leifer, D., D. Krainc, Y.-T. Yu, J. McDermott, R.E. Breitbart, J. Heng, R.L. Neve, B. Kosofsky, B. Nadal-Ginard, and S.A. Lipton. 1993. MEF2C, a MADS/MEF2-family transcription factor expressed in a laminar distribution in cerebral cortex. Proc. Natl. Acad. Sci. 90: 1546-1550.

Logan, M.L. and T.J. Mohun. 1993. Induction of cardiac muscle differentiation in isolated animal pole explants of Xenopus laevis embryos. Development 118: 865-875.

Martin, J.F., J.J. Schwarz, and E.N. Olson. 1993. Myocyte enhancer factor (MEF) 2C: A tissue-restricted member of the MEF-2 family of transcription factors. Proc. Natl. Acad. Sci. 90: 5282-5286.

McDermott, J.C., M.C. Cardoso, Y. Yu, V. Andres, D. Leifer, D. Kraine, S.A. Lipton, and B. Nadal-Ginard. 1993. hMEF2C gene encodes skeletal muscle- and brain-specific transcription factors. Mol. Cell. Biol. 13: 2564-2577.

Melton, D.A., P.A. Krieg, M.R. Rebagliati, T. Maniatis, K. Zinn, and M.R. Green. 1985. Efficient in vitro synthesis of biologically active RNA and RNA hybridisation probes from plasmids containing a bacteriophage SP6 promoter. Nucleic Acids Res. 12: 7035-7056.

Mohun, T., S. Brennan, N. Dathan, S. Fairman, and J. Gurdon. 1984. Cell type-specific activation of actin genes in the early amphibian embryo. Nature 311: 716-721.

Mohun, T.J., N. Garrett, F. Stutz, and G. Spohr. 1988. A third striated muscle actin gene is expressed during early development in the amphibian Xenopus laevis. J. Mol. Biol. 202: 67-76.

Molkentin, J.D. and B.E. Markham. 1993. Myocyte-specific enhancer-binding factor (MEF-2) regulates alpha-cardiac myosin heavy chain gene expression in vitro and in vivo. 1 . Biol. Chem. 268: 19512-19520.

Nakatsuji, Y., K. Hidaka, S. Tsujino, Y. Yamamoto, T. Mukai, T. Yanagihara, T. Kishimoto, and S. Sakoda. 1992. A single MEF-2 site is a major positive regulatory element required for transcription of the muscle-specific subunit of the human phosphoglycerate mutase gene in skeletal and cardiac muscle cells. Mol. Cell. Biol. 12: 4384-4390.

Navankasattusas, S., H. Zhu, A.V. Garcia, S.M. Evans, and K.R. Chien. 1992. A ubiquitous factor (HF-1a) and a distinct muscle factor (HF-1b/MEF-2) form an E-box-independent pathway for cardiac muscle gene expression. Mol. Cell. Biol. 12: 1469-1479.

Nieuwkoop, P. and J. Faber. 1956. Normal table of Xenopus laevis (Daudin). North-Holland, Amsterdam, The Netherlands. 
Olson, E.N. 1992. Interplay between proliferation and differentiation within the myogenic lineage. Dev. Biol. 154: 261272.

Parmacek, M.S., A.J. Vora, T. Shen, E. Barr, F. Jung, and J.M. Leiden. 1992. Identification and characterization of a cardiac-specific transcriptional regulatory element in the slow/ cardiac troponin C gene. Mol. Cell. Biol. 12: 1967-1976.

Pollock, R. and R. Treisman. 1990. A sensitive method for the determination of protein-DNA binding specificities. Nucleic Acids Res. 18: 6197-6204.

1991. Human SRF-related proteins: DNA-binding properties and potential regulatory targets. Genes \& Dev. 5: 2327-2341.

Rashbass, J., M.V. Taylor, and J.B. Gurdon. 1992. The DNAbinding protein E12 co-operates with XmyoD in the activation of muscle-specific gene expression in Xenopus embryos. EMBO J. 11: 2981-2990.

Sargent, M.G. and M.F. Bennett. 1990. Identification in Xenopus of a structural homologue of the Drosophila gene snail. Development 109: 967-973.

Sater, A.K. and A.G. Jacobson. 1989. The specification of heart mesoderm occurs during gastrulation in Xenopus laevis. Development 105: 821-830.

- 1990. The restriction of the heart morphogenetic field in Xenopus laevis. Dev. Biol. 140: 328-336.

Staden, R. 1982. Automation of the computer handling of gel reading data produced by the shotgun method of DNA sequencing. Nucleic Acids Res. 10: 4731-4751.

1984. Graphic methods to determine the function of nucleic acid sequences. Nucleic Acids Res. 12: 521-538.

Taylor, M.V., J.B. Gurdon, N.D. Hopwood, N. Towers, and T.J. Mohun. 1991. Xenopus embryos contain a somite-specific, MyoD-like protein that binds to a promoter site required for muscle actin expression. Genes \& Dev. 5: 1149-1160.

Wentworth, B.M., M. Donoghue, J.C. Engert, E.B. Berglund, and N. Rosenthal. 1991. Paired MyoD-binding sites regulate myosin light chain gene expression. Proc. Natl. Acad. Sci. 88: 1242-1246.

Winter, B., H. Klapthor, K. Wiebauer, H. Delius, and H.H. Arnold. 1985. Isolation and characterization of the chicken cardiac myosin light chain (1-2a) gene. J. Biol. Chem. 260: 44784483.

Yee, S.P. and P.W. Rigby. 1993. The regulation of myogenin gene expression during the embryonic development of the mouse. Genes \& Dev. 7: 1277-1289.

Yu, Y.T., R.E. Breitbart, L.B. Smoot, Y. Lee, V. Mahdavi, and B. Nadal-Ginard. 1992. Human myocyte-specific enhancer factor 2 comprises a group of tissue-restricted MADS box transcription factors. Genes \& Dev. 6: 1783-1798.

Zhou, M.D., S.K. Goswami, M.E. Martin, and M.A. Siddiqui. 1993. A new serum-responsive, cardiac tissue-specific transcription factor that recognizes the MEF-2 site in the myosin light chain-2 promoter. Mol. Cell. Biol. 13: 1222-1231.

Zhu, H., A.V. Garcia, R.S. Ross, S.M. Evans, and K.R. Chien. 1991. A conserved 28-base-pair element (HF-1) in the rat cardiac myosin light-chain-2 gene confers cardiac-specific and alpha-adrenergic-inducible expression in cultured neonatal rat myocardial cells. Mol. Cell. Biol. 11: 2273-2281.

Zhu, H., V.T. Nguyen, A.B. Brown, A. Pourhosseini, A.V. Garcia, M. van Bilson, and K.R. Chien. 1993. A novel, tissuerestricted zinc finger protein (HF-lb) binds to the cardiac regulatory element (HF-1b/MEF-2) in the rat myosin lightchain 2 gene. Mol. Cell. Biol. 13: 4432-4444. 


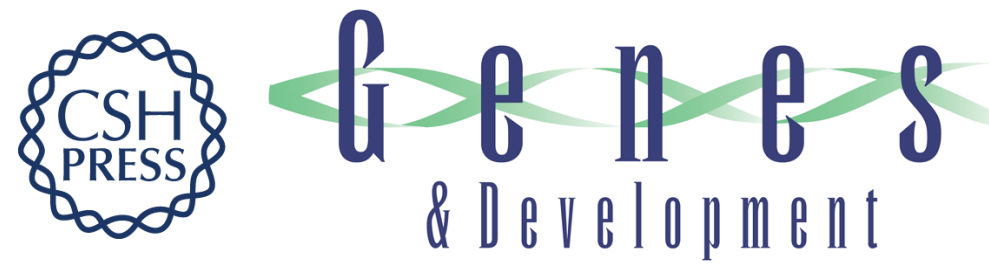

\section{The RSRF/MEF2 protein SL1 regulates cardiac muscle-specific transcription of a myosin light-chain gene in Xenopus embryos.}

A E Chambers, M Logan, S Kotecha, et al.

Genes Dev. 1994, 8:

Access the most recent version at doi:10.1101/gad.8.11.1324

References This article cites 56 articles, 37 of which can be accessed free at:

http://genesdev.cshlp.org/content/8/11/1324.full.html\#ref-list-1

License

Email Alerting

Service

Receive free email alerts when new articles cite this article - sign up in the box at the top right corner of the article or click here.

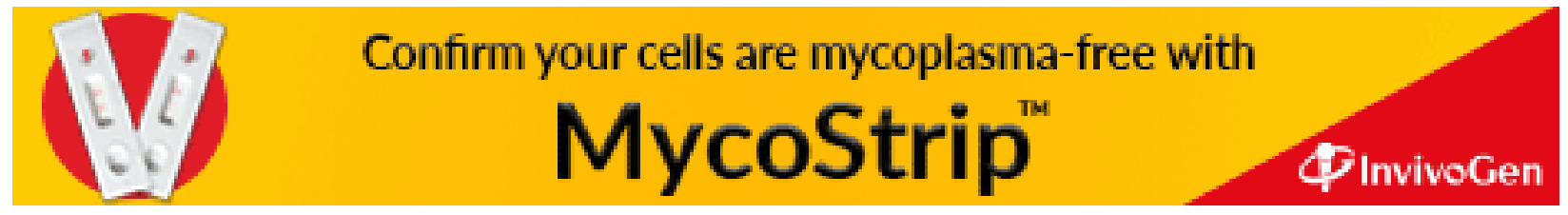

